\title{
Development of laser-induced grating spectroscopy for underwater temperature measurement in shock wave focusing regions
}

\author{
Ardian B. Gojani, ${ }^{* a}$ Paul M. Danehy, ${ }^{b}$ David W. Alderfer, ${ }^{b}$ Tsutomu Saito, ${ }^{a}$ Kazuyoshi Takayama, ${ }^{a}$ \\ ${ }^{a}$ Interdisciplinary Shock Wave Research Center, Institute of Fluid Science, Tohoku University, \\ 2-1-1 Katahira, Aoba, Sendai 980-8577, Japan \\ ${ }^{\mathrm{b}}$ NASA Langley Research Center, Hampton, Virginia 23681-2199 USA
}

\begin{abstract}
In Extracorporeal Shock Wave Lithotripsy (ESWL) underwater shock wave focusing generates high pressures at very short duration of time inside human body. However, it is not yet clear how high temperatures are enhanced at the spot where a shock wave is focused. The estimation of such dynamic temperature enhancements is critical for the evaluation of tissue damages upon shock loading. For this purpose in the Interdisciplinary Shock Wave Research Center a technique is developed which employs laser induced thermal acoustics or Laser Induced Grating Spectroscopy. Unlike most of gasdynamic methods of measuring physical quantities this provides a non-invasive one having spatial and temporal resolutions of the order of magnitude of $1.0 \mathrm{~mm}^{3}$ and $400 \mathrm{~ns}$, respectively. Preliminary experiments in still water demonstrated that this method detected sound speed and hence temperature in water ranging $283 \mathrm{~K}$ to $333 \mathrm{~K}$ with errors of $0.5 \%$. These results may be used to empirically establish the equation of states of water, gelatin or agar cells which will work as alternatives of human tissues.
\end{abstract}

Keywords: Laser induced grating spectroscopy, underwater shock wave, temperature measurement, medical application, equation of state.

\section{INTRODUCTION}

The nearly instantaneous change of fluid velocities and pressures caused by shock waves is being utilized for various medical applications. Shock wave-assisted lithotripsy is widely used by medical doctors for non-invasive disintegration of kidney stones and treatments of gall bladder diseases. For these purposes, underwater shock waves are focused in a localized region inside human body, in a volume as small as $3 \mathrm{~mm}$ in diameter and $11 \mathrm{~mm}$ in length, with the overpressure range from 10 to $100 \mathrm{MPa}$, and with a typical pulse width from hundreds of nanoseconds up to about 50 microseconds ${ }^{1}$. Despite the successful and habitual application of extracorporeal shock-wave lithotripsy (EWSL) which is used for the treatment of about $70 \%$ of all kidney stone cases, the detailed physics of the process still is not fully understood. As an illustration of this is the fact that the complete computational stimulation of ESWL was only completed in $1999^{2}$. It has also been noticed that shock waves applied in lithotripsy can permanently damage some of the healthy kidney tissue, but the mechanism of this is not fully explained. It is believed that two effects cause the damage, those being the dissipation of shock wave energy and the jet induced from cavitations bursts ${ }^{3}$. Recently shock waves have become an active research field for delivery of small particles ${ }^{4}$. If the shock wave is loaded in a tissue the living cells become permeable for a short time, thus enabling delivery of drugs and other macromolecules. The process of cell perforation and other mechanisms of shock interaction with cell in microscopic level are poorly understood. On the other hand, innovative techniques are being developed for the treatment of cancerous tumors using the method of hyperthermia. So far, progress has been reported for facilitation of ultrasound technology ${ }^{5}$, while work on applying shock waves is being proposed. This method consists on locally elevating the temperature of the target region to $42-45{ }^{\circ} \mathrm{C}$ for a period of $30-60$ minutes, and then applying radiation therapies. Tissue treatment with hyperthermia enhances clinical response.

Underwater shock waves are generated and focused using various methods, in the basis of which lays the immediate release of pulsed energy and its coalescence in a secondary focal point of a reflector. Available methods for shock generation include point electrical breakdown in water, explosion of micro-explosives, pump laser beam focusing, and 
actuation of piezo-ceramic arrays. The generated shock waves will be reflected from the wall of the reflector, which usually has the shape of a truncated cavity of an ellipsoid of revolution. After being reflected, shock waves focus at the second focal point reaching peak pressures, which are utilized for medical purposes.

In order to exactly describe the hydrodynamic processes of underwater shock wave propagation and focusing the equation of state for water should be correctly determined in the range at which underwater shocks are applied. Existing equations of state are constructed mainly for relatively strong shock waves in the range of hundreds and thousands of $\mathrm{MPa}^{6}$, so can not be used for describing underwater shock waves used in medicine. Hence, estimation of degree of temperature enhancement behind relatively weak underwater shock waves is of a paramount importance. Temperature increase following underwater shock wave propagation is due to entropy increase and isentropic compression? ${ }^{7}$.

Conventionally temperatures are measured by using thermometers, thermocouples, and resistance temperature detectors, inserted into the fluid. However, their response time is generally to slow to measure the rapid temperature change of a shocked fluid, and their insertion disturbs the flow. On the other hand, infrared and optical pyrometers can measure temperatures non-intrusively, but they are useful only at high temperatures. Therefore, a non-intrusive, temperature measurement technique with high spatial and temporal resolution is needed. Recently a new laser induced transient grating spectroscopic (LIGS) technique, also termed laser-induced thermal acoustics (LITA) has received attention as a quantitative probe of thermophysical medium properties.

The physical principles of the laser-induced grating technique lies in the following: two pulsed laser beams of equal intensity and equal wavelength intersect at a shallow angle in test volume. In the intersection region these beams interfere, thus creating an electric and thermal field grating by molecular mechanisms of electrostriction and thermalization, which results in a density, and hence, refractive index grating. The evolution of this grating is examined by a continuous interrogation beam, which is directed at the Bragg angle, because it has to satisfy the phase matching condition. Part of the probe beam that passes through grating is coherently scattered into a signal beam. Since the density grating evolves with time and the signal beam intensity is proportional to the instantaneous density grating modulation depth, the signal allows us to observe the grating evolution.

Laser-induced spectroscopy using transient gratings ${ }^{8}$ has been practiced for the study of acoustic phonons and excited-state phonon interaction in molecular crystals ${ }^{9,10}$, to measure thermal diffusivity and speed of sound in $p$-dimethylaminobenzaldehyde disolved in water $^{11}$, as well as to measure the sound velocity and the attenuation coefficient of biological material, including vitreous, eye lens, and blood for some animals, at room temperature ${ }^{12,13}$. Laser-induced spectroscopy has found most of its applications for diagnostics in gas phase, and initially it was developed for measurements of hypersonic flows $\mathbf{s}^{14,15}$. Contributions of thermal and population grating mechanisms to the obtained signal have been reported ${ }^{16}$, as well as contribution of electrostriction ${ }^{17}$. The present work confirms the results for inferring the temperature from the measurement of sound speed of quiescent water ${ }^{18}$ and tries to demonstrate technique's capacity for evaluation of temperature enhancement due to underwater shock waves.

\section{THEORETICAL CONSIDERATION}

Laser-induced grating spectroscopy has grown after conjunction of methods of optical four-wave mixing ${ }^{8}$ and methods of dynamic light scattering ${ }^{19}$. The first method covers the creation of an interference grating in a medium by the use of two coincident light beams. The grating formation process is called pumping, and represents the spatial modulation of the medium where the grating occurs. This grating volume is probed by a third laser beam, which directed in a particular direction. The third beam, termed the source, will diffract at the grating volume, thus creating a fourth coherent signal. The fourth beam will rise due to nonlinear properties of matter. The signal in four-wave mixing is detected and analyzed, thus revealing information about grating and its evolution in time. Dynamic light scattering presents the intensity of the scattered light by a fluid, dependent on time. After an incident electromagnetic field is applied to a volume of fluid, it will force a dipolar moment in the molecules, parallel to the direction of the incoming electromagnetic wave. These internal dipole moments will give rise to a re-emission of light. In case of homogeneous distribution of dipoles, light will add up only in the incident direction, due to phase cancellation in other directions. However, if medium is disturbed in microscopic level, then these random fluctuations will cause light to scatter. Moreover, since the molecules are in permanent Brownian motion, the scattered light will be even frequency shifted, due to Doppler effect. Fluctuations can be described by macroscopic thermodynamic properties, thus neglecting the molecular point of view. Thus, medium disturbances can be temperature and entropy fluctuations, disturbances of chemical nature, concentration, fluctuations in orientation and vibration of molecules, and microscopic acoustic waves. These fluctuations will decay in time to a 
macroscopic equilibrium, allowing us to use hydrodynamic equations for investigation of changes of fluctuations with time. Consequently, the scattered signal will encode transport properties of the fluid, such as thermal diffusivity, diffusion coefficient for mixtures, sound absorption coefficient, and speed of sound.

Gratings for laser-induced phenomena are created by interference of two laser light beams. In experiments, gratings are usually generated by division of a laser beam into two and then arranged to intersect at their foci. If beams intersect at an angle $\theta$, then grating will have the shape of a Gaussian ellipsoid with dimensions $l=d / \sin \theta, h=d / \cos \theta$, where $d$ is the beam waist diameter. The grating periodicity (fringe spacing) will be

$$
\Lambda=\frac{\lambda_{p}}{2 \sin \left(\frac{\theta}{2}\right)},
$$

where $\lambda_{p}$ is the wavelength of the pumping laser light.

Spatially periodic illumination of the medium caused by the interference of the two incident laser beams causes spatially periodic changes of some of the physical properties of the medium, i.e. temperature, molecular orientation, and other properties irrelevant to the signal for our experiment. The material will respond to these changes by two molecular mechanisms, thermalization and electrostriction.

Thermalization describes the process of absorption of light energy by fluid molecules and its conversion into thermal energy by means of collisions. When the molecules in the target medium contain energy level differences resonant to the input laser light wavelength, the molecules will absorb the input photons and be excited to a higher energy level. The excited molecules collide with other molecules. When the collision is non-elastic, the absorbed laser energy is converted to thermal energy. The local temperature increase produced by thermalization can be approximated as ${ }^{20}$

$$
\Delta T=\phi \frac{K}{\rho c_{p}}\left(2 I \tau_{p}\right),
$$

where $\Delta T$ is the temperature modulation, $K$ is the absorption coefficient, $\tau_{p}$ is the pump laser pulse width, $c_{p}$ is the isobaric specific heat coefficient, $\rho$ is density, and $I$ is the light intensity. The dimensionless factor $\phi$ is fraction of absorbed energy transferred by collisions to the surrounding medium. Electrostriction is the tendency of polarizable molecules to move under the action of a potential filed caused by the interaction of an optically induced dipole moment and optical filed, which means that material becomes compressed, or rarefied, under the presence of an electric field ${ }^{21}$. The electrostrictive force is actually the consequence of the maximization of potential energy. When an electric field is applied, the polarizable molecules are first polarized. Then they are accelerated towards the regions of high field intensity gradient. If the driving force is rapid, the acceleration is impulsive, and a local density disturbance will be produced. Electrostriction will not add heat to the medium, while thermalization, in accordance with equation (2) will increase the temperature of the medium in the order of less than 0.1 degree. By this, the non-intrusiveness of the technique is assured.

These two processes affect the medium by changing its susceptibility and by generating acoustic waves. Change of susceptibility of the medium means that a diffraction grating is formed; therefore the medium can be probed by diffraction of a light wave. On the other hand, acoustic wave modulates the diffracted light. Acoustic waves propagate by diffusion and induce density grating, and their amplitude decays at the acoustic damping rate. The frequency of the induced density field modulation equals the Brillouin frequency and its dynamic behavior is described by the linear hydrodynamic equations ${ }^{22}$.

Evolution of the transient grating is observed by probing it with a continuous laser light. Nonuniformities in fluid scatter incident light, and if these nonuniformities are of isobaric and isentropic nature, then Reyleigh and Brillouin scattering occurs. In the case of small fluctuations (nonuniformities), the scattered signal is going to be incoherent, therefore of weak intensity. However, in laser-induced grating spectroscopy experiments fluctuations are coherent due to laser induction, which results in coherent scattered signal. This signal is many orders of magnitude stronger, although its spectrum is preserved. Three incident laser lights (two pumping beams and one probing beam) are delivered into the test 
section, and a phase matched fourth beam, the signal, is generated. The direction of the signal will close an angle $\Phi$ with the probing beam according to Bragg's law, i.e.

$$
\frac{\lambda_{p}}{2 \sin \frac{\theta}{2}}=\frac{\lambda_{s}}{2 \sin \frac{\Phi}{2}},
$$

where $\lambda_{s}$ is the wave length of the probing or signal beam.

\section{EXPERIMENTAL SETUP}

A schematic diagram of the experimental setup is shown in Fig. 1. Frequency-doubled $(532 \mathrm{~nm})$ output of a pulsed Nd:YAG laser with $\sim 10 \mathrm{~ns}$ pulse duration was divided into two equal parts and sent through transmitting optics at the testing medium. Laser energy was $60 \mathrm{~mJ} /$ pluse and it had a $10 \mathrm{~Hz}$ repetition rate. This light was used as the pumping beam for the grating generation. The transient laser induced grating is probed by the beam of a continuous wave $\mathrm{Ar}^{+}$ laser. This laser's output was a $488 \mathrm{~nm}$ wavelength light with power of approximately $0.4 \mathrm{~W}$.

A set of high-reflection mirrors and prisms were used as a transmitting optics, to direct the beams at the test section. Prior to the transmitting optics, a washer $w$ was inserted into the path of the pump beam to make the focus spot size larger in the direction of propagation of acoustic waves. This was done in order to increase the number of acoustic waves, in the cost of reduction of the spatial resolution. The pump beam was split into two equal parts with a beam splitter $b s$. An acousto-optics modulator (AOM) was used to deflect the probe beam into the test section. One reason for using the AOM is that the laser light is going to be focused in a small spot inside the water, and this can heat the water locally, as well as make the water spot act like a lens, thus misaligning the beams. Another reason for using the AOM was to prevent scattered light from the continuous wave $\mathrm{Ar}^{+}$laser from being collected by the signal detector between laser pulses, to avoid damaging the detector. Pulses of the probe beam were $30 \mu$ s long at $10 \mathrm{~Hz}$, and they were synchronized with the pump beam through the pulse generator.

The geometrical arrangement of the beams to meet the phase matching condition was checked with a template inserted at the place where the lens was put at the time of the measurements, and another template was located at a distance approximately $4 \mathrm{~m}$ further, at the end of the laboratory room. In order to trace the path of the signal, $10 \%$ of the reading beam (not shown in Fig. 1), was parted and phase matched with the other three beams. This simulated signal beam was followed up to the detection system.

The pump beams were crossed and focused at the test volume using a thin plano-convex lens, $l_{l}$. Most measurements were done with a lens with $750 \mathrm{~mm}$ focal length, but a $300 \mathrm{~mm}$ focal length lens was used for a set of measurements, too. Approximately $20 \mathrm{dl}$ of water was put in a fish tank. In order to reduce the intensity of scattered light the fish tank was covered with black sheets. The water was heated with a series of ordinary coffee cup heaters and its temperature was monitored with a thermocouple, TC, which was located a few millimeters apart from the intersection region of the laser beams, at the same height. Beams which emerged from the test section were stopped by two beam dumps, while the signal was sent through a set of apertures. The signal, being generated by a nonlinear process, is of a very weak intensity, usually of the order of a millionth or less compared to the probe beam intensity. In order to detect these weak signals photomultiplier tubes (PMT) are used. The advantage of the signal is its coherent nature; hence most of the generated signal is collected.

The signal was led through the collecting optics, which was compound of a Pellin-Broca prism, PBP, an aperture (iris) $i$, a collecting lens, $l_{2}$, pinhole, $p h$, a laser line bandpass filter, a collimating lens, $l_{3}$, and through fiber optics, FO, to PMT, elapsing approximately $2.5 \mathrm{~m}$ distance. The aim of using PBP was so the light of different wavelength would be dispersed and the signal would be separated from undesirable noise of different wavelength than the wavelength of the signal, which is the same as the wavelength of the probing light. The collecting lens, pinhole and collimating lens make the so called spatial filter set. A spatial filter is an aperture placed at the focal point of a telescopic lens pair. Since the background scatterings are incoherent, only background noise sharing the same path with signal beam will be focused at the center of the aperture. Therefore, a spatial filter allows only very small solid angle aligned with the signal path to pass through. This not only protects the photon detector, but also suppresses the background noise which makes the signal undistinguishable. The pinhole used in experiments had a $100 \mu \mathrm{m}$ aperture. The line bandpass filter was used to 
selectively transmit only the signal, discriminating over laser fluorescence that went on the same path as the signal. This filter provided protection to the PMT from strong photon flux, as well.

PMT used was a Hamamatsu H6779/H6780 with at least $500 \mathrm{MHz}$ signal bandwidth. This type of PMT satisfies both conditions for reading the signal, i.e. they are sensitive and fast responding. Sample signals were recorded using1 GS/s, four channel, $2 \mathrm{GHz}$ digital storage oscilloscope. Triggering was done using a SRS model DG535 digital delay/pulse generator, which was connected to the control panel of the Nd:YAG laser's flashlamps, AOM, and one of the four channels of the digital oscilloscope. Connections were done using BNC connectors.

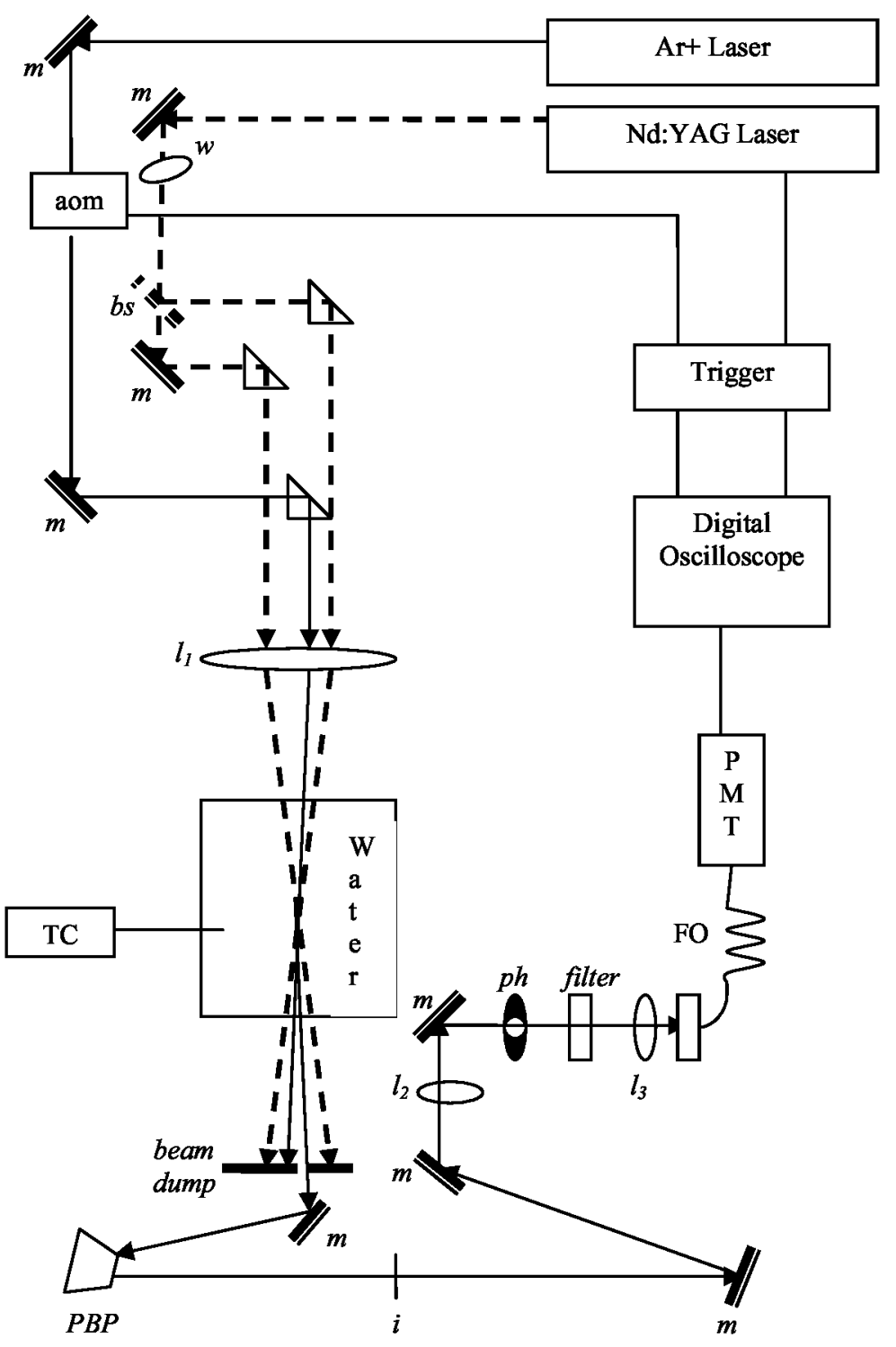

Fig. 1. Schematic setup of the experiment.

\section{RESULTS}

Selected results of the experiments are given in the following. Although laser-induced grating spectroscopy makes possible measurements of various thermophysical and chemical properties of fluids, these results are obtained only with the aim of evaluating the temperature through measurement of the speed of sound. Data for a single measurement were compiled from 500 collected points, each recorded in $1.6 \mathrm{~ns}$. In these experiments, focused beams were employed, 
providing strong signals, while the option for high spatial resolution was traded with realization of wide interaction regions, which resulted in many oscillations, consequently, into more accurate frequency determination.

Different examples of the signals and their respective spectra are shown in Figures 2, 3 and 4. Fig. 2a shows the signals obtained from a 10 shot average and a single shot measurement. In order to compare the frequencies of the signals, their respective spectra are shown in Fig. $2 b$. Figures $3 a$ and $3 b$ give the signals and their respective spectra for two different intersection angles of the beams. The long curve corresponds to the arrangement for an angle of incidence $\sim 1.5^{\circ}$, while the short curve corresponds to arrangement for an angle of incidence $\sim 3.5^{\circ}$. Angles were changed by inserting focusing lenses with different focal lengths, $750 \mathrm{~mm}$ and $300 \mathrm{~mm}$. Despite the fact that the beam directions were not altered at targeting the template during the change of the lenses, small adjustments were necessary for obtaining a strong signal. Therefore, precise determination of the intersection angle is an obstacle to fully exact results. Finally, Fig. 4a shows signals at different temperatures, while Fig. $4 \mathrm{~b}$ gives their respective spectra. Six temperatures were investigated. In all figures, signals due to electrostriction and thermalization are observed.

Independent parameters that enter this measurement technique were angle of intersection of the pumping beams, $\theta$ in equation (1), and diameter of pump and probe laser. Other parameters, such as laser energy, laser pulse duration, etc., were fixed based on equipment specifications. The angle of intersection was not adjusted by redirecting laser beams, because that would involve tedious work, but it was altered by changing lenses, thus changing focal length. Diameters of laser pulses are not critical for evaluation of speed of sound, but they influence the spatial resolution of the probing volume.

The intersection angle directly influences fringe periodicity, $\Lambda$, which is related to the speed of sound as the acoustic wave wavelength. But, there isn't any reliable method for determination of this angle. In calculating the fringe periodicity two ways can be followed: measuring the distance between parallel pump beams, $X$, and then obtaining $\theta$ from the relation $\theta=2 \tan ^{-1}\left(\frac{X}{2 f_{l}}\right)$, where $f_{l}$ is the focal length, or by calibrating the measurements at a known temperature, and from there to calculate the fringe periodicity. For the calculations in the following, the second method was adopted, although even the first method gave reasonable results. The following results are calculated with the value of the intersection angle in air $\sim 1.8^{\circ}$. In calculating this value, refraction of light when entering and exiting water medium was not calculated. It is believed that the error inherited in determination of $\theta$ is $\pm 1 \%$. The value for fringe periodicity can be calculated from equation (1) and we get $\Lambda=34 \pm 0.5 \mu \mathrm{m}$. The grating volume, which defines the spatial precision, is calculated to be $(0.3 \times 0.7 \times 5) \mathrm{mm}^{3}$.

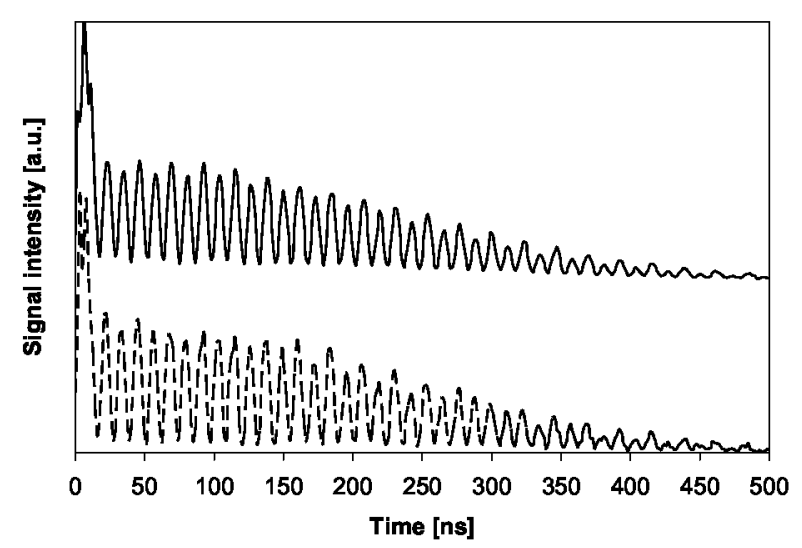

a)

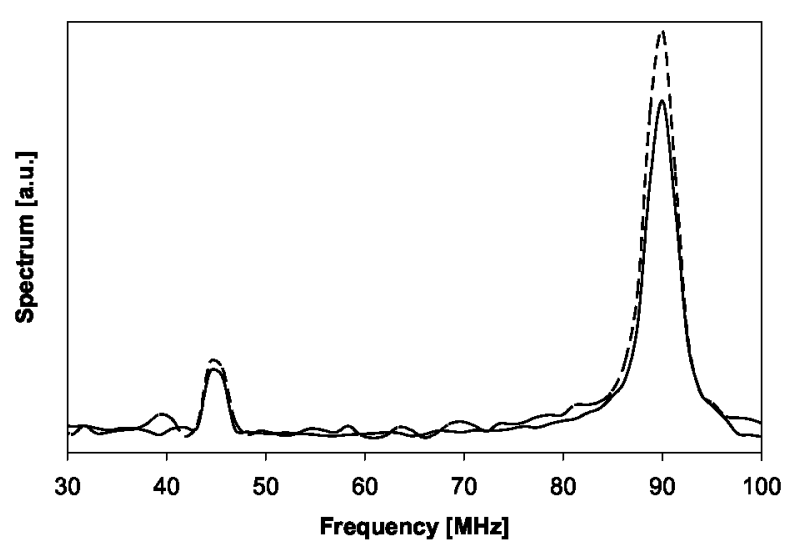

b)

Fig. 2. a) Comparison of 10 shot average signals (dashed line) and single shot signal (full line), shifted for clarity; b) Spectra of the 10 shot average signals (dashed line) and single shot signal (full line). 


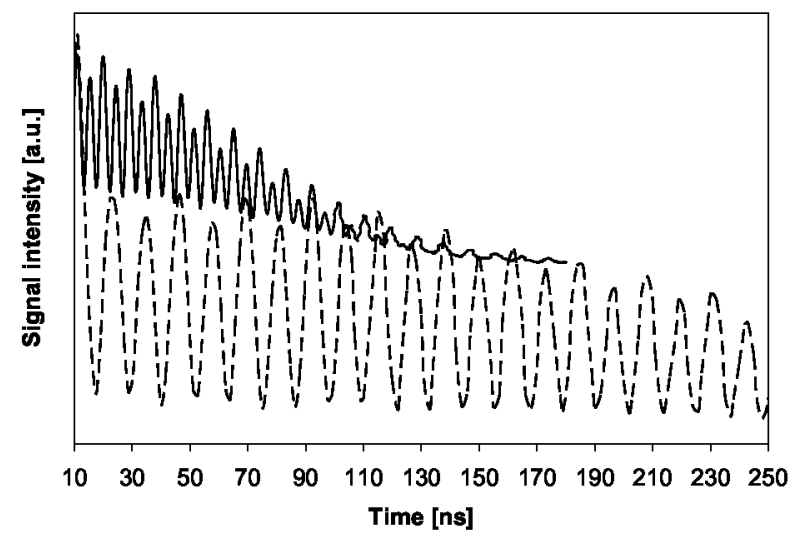

a)

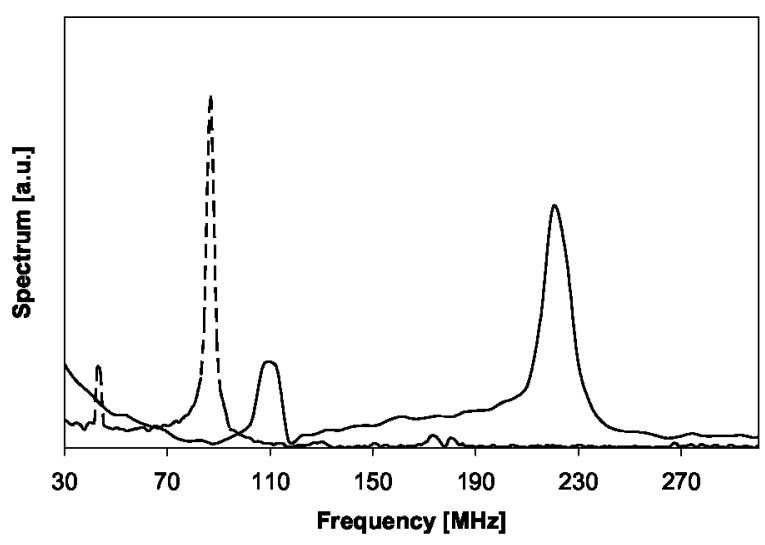

b)

Fig. 3. a) Comparison of signals for the angles of incidence $\sim 1.5^{0}$ (dashed line) and $\sim 3.5^{0}$ (full line), shifted for clarity; b) Spectra of the signals for arrangements with angles of incidence $\sim 1.5^{\circ}$ (dashed line) and $\sim 3.5^{\circ}$ (full line).

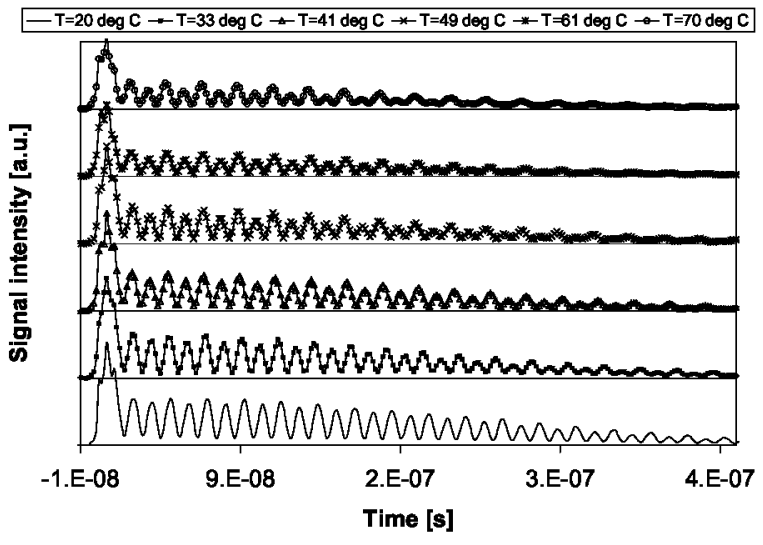

a)

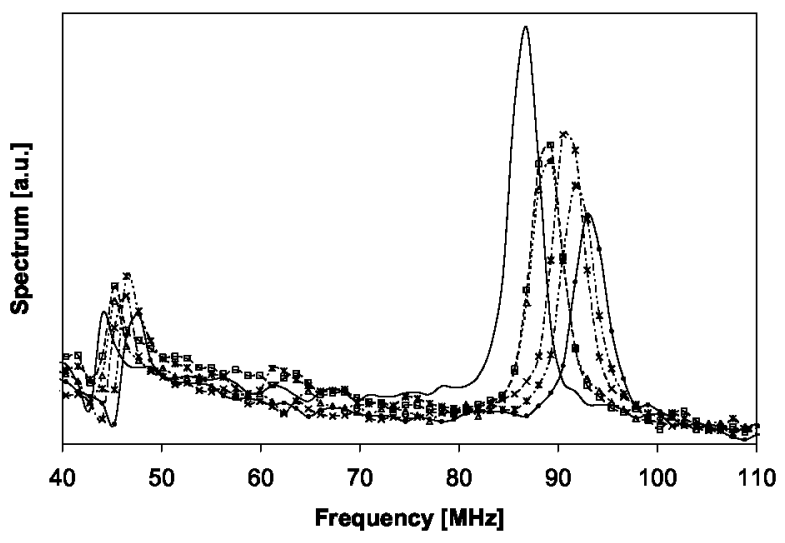

b)

Fig. 4. a) Comparison of signals in water with different temperatures, shifted for clarity; b) Spectra of the signals for different temperatures of water.

\begin{tabular}{cccc}
\hline$T(\mathrm{TC})\left[{ }^{\circ} \mathrm{C}\right]$ & $v_{t h}[\mathrm{MHz}] \pm 0.5 \%$ & $v_{e l}[\mathrm{MHz}] \pm 0.5 \%$ & $c$ (calulated) $[\mathrm{m} / \mathrm{s}]$ \\
\hline 20.6 & 44.2 & 86.7 & 1484.9 \\
33.3 & 45.3 & 88.9 & 1522.9 \\
41.8 & 45.4 & 89.1 & 1526.3 \\
50.1 & 46.4 & 90.9 & 1558.5 \\
62 & 46.7 & 91.8 & 1571.4 \\
70.5 & 47.5 & 93.1 & 1595.9 \\
\hline
\end{tabular}

Table 1. Values for temperature, $T$, read at thermocouple, for frequency due to thermalization, $v_{t h}$, amd due to electrostriction, $v_{e l}$, obtained from spectra of the signal, and calculated speed of sound, $c$. 


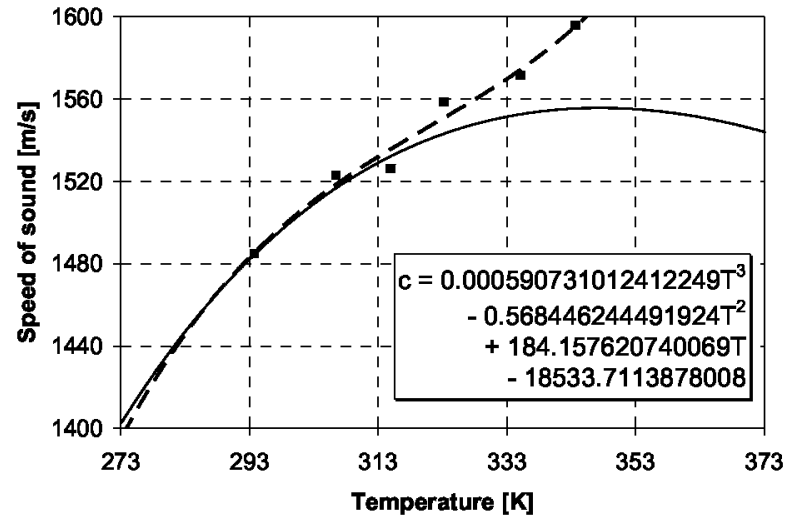

Fig. 5. Relation between temperature and speed of sound of water in atmospheric pressure. Squares correspond to temperatures obtained by LIGS measurements, dashed line represents the obtained relation $c=c(T)$.

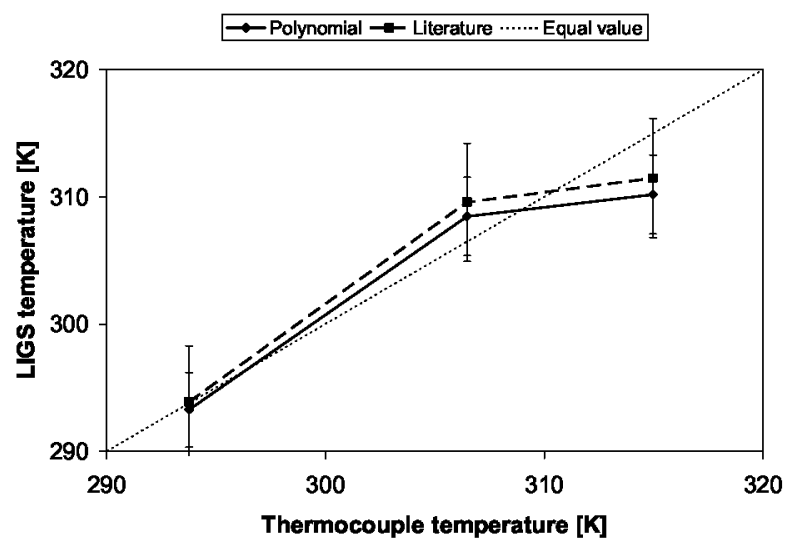

Fig. 6. Comparison of temperature measurement inferred by LITA with thermocouple temperature reading.

Speed of sound is calculated by using equation $c=\Lambda v$, where $v$ is the frequency, deduced from the spectra of the signal (Fig. 2b, 3b, 4b). The signal provides the information about the frequency with an accuracy of $\pm 0.5 \%$. Spectra of the signal were obtained by processing data with Fast Fourier Transform tool of MS Excel and the values of frequencies are given in Table 1. Here, speed of sound is calculated and its polynomial relation to the temperature is given. Polynomial is calculated using least squares fit.

From an initial survey of Fig. 5. it can be concluded that in the range of temperatures between $20^{\circ} \mathrm{C}$ and $45^{\circ} \mathrm{C}$ a good correspondence between the speed of sound and temperatures exist. For measurements when water was heated over 45 ${ }^{\circ} \mathrm{C}$, the values of the speed of sound drastically exceed the maximum of the water sound speed. Table 2 summarizes the measured values of speed of sound and temperature, as well as the corresponding values from thermocouple and literature $^{23}$. Comparison of temperature measurement by thermocouple and LIGS technique is shown in Fig. 6. Bars present an error of $1 \%$.

\begin{tabular}{cccc}
\hline $\begin{array}{c}\text { LIGS speed of } \\
\text { sound }[\mathrm{m} / \mathrm{s}]\end{array}$ & $\begin{array}{c}\text { Thermocouple } \\
\text { temperature }\left[{ }^{\circ} \mathrm{C}\right]\end{array}$ & $\begin{array}{c}\text { Temperature from } \\
\text { polynomial }\left[{ }^{\circ} \mathrm{C}\right]\end{array}$ & $\begin{array}{c}\text { Temperature from } \\
\text { literature }\left[{ }^{\circ} \mathrm{C}\right]\end{array}$ \\
\hline 1484.87 & 20.6 & 20.1 & 20.7 \\
1522.89 & 33.3 & 35.3 & 36.4 \\
1526.28 & 41.8 & 37 & 38.3 \\
\hline
\end{tabular}

Table 2. Comparison of temperatures for a fixed value of the speed of sound, obtained by LIGS.

It is interesting to note the results that can be deduced from Figures 2, 3, and 4. The first case demonstrates the fact that LITA technique gives good single shot results. As it is seen from Fig. 2b, the frequencies of the single shot signal and 10 shots averaged signal are the same. Therefore, LITA is suitable for transit phenomena that change rapidly. Since the time resolution of LITA technique for the current setup is approximately 200 nanoseconds, and the underwater shock waves have rise time of the order of microseconds, LITA technique is applicable for underwater measurements in terms of time 
resolution. The other case compares the signals when angles of incidence are different. It can be deduced that the more acute the angle, the better is the time resolution and more oscillations are obtained.

\section{CONCLUSIONS}

Laser-induced grating spectroscopy was applied for quiescent water temperature evaluation by measuring the speed of sound, at the range from 20 up to $70^{\circ} \mathrm{C}$. The signal grew as a diffraction of a probe laser beam by a transient volume grating, generated by interference of two pump laser beams. Two distinct processes that contribute to the signal, namely thermalization and electrostriction, were theoretically considered and observed by experiment. At the grating volume, an acoustic wave is generated, which in turn modulates the signal. From the geometrical arrangement of the experimental setup, volume grating dimensions were calculated, resulting in spatial resolution of the technique of the order of hundreds of micrometers. Time resolution of the measurement is less than a half of a microsecond. It has been shown that steeper angles of intersection of the pump beams result in a better temporal and spatial resolution. Furthermore, based on the angle of intersection, the frequency of the signal can be tuned two orders of magnitude in megahertz region.

The current work was focused on evaluation of the adiabatic speed of sound from frequency analysis of the oscillating part of the LIGS signal. Relations for speed of sound and temperature of water was used to infer the water temperature. Values for temperature were compared with measurements done by a thermocouple. Conceptually, it has been successfully shown the dependence of speed of sound with temperature by using laser induced grating spectroscopy. Nevertheless, obtained results were accurate within $1 \%$ error in the range of temperatures higher than $20^{\circ} \mathrm{C}$ and lower than $45^{\circ} \mathrm{C}$. In water temperatures higher than $45^{\circ} \mathrm{C}$, the obtained signal gave big discrepancies in inferring the speed of sound with temperature. This is due to the fact that a wide domain of temperatures corresponds to a certain speed of sound in that region.

It has been shown that LIGS provides reliable single-shot point measurements with high spatial and temporal accuracy, without obtruding in the medium. Since it is estimated that the temperature enhancement behind underwater shock waves applied in medicine would not exceed $50^{\circ} \mathrm{C}$, LIGS technique is applicable for underwater shock wave diagnostics. Still, the effects of two parameters in the signal should be investigated: pressure gradient and temperature gradient. High pressures do not posses an obstacle for generating the signal, but the effect of the pressure gradient is unknown. However, at high pressures the sound speed depends on both temperature and pressure, complicating the analysis. On the other hand, an immediate task is finding the solutions for measurements in the temperature range higher than $45^{\circ} \mathrm{C}$. It is intended to try LIGS experiments in other liquids, because water features several "unusual" properties compared to a number of liquids. For example, the change of the speed of sound with the change of temperature in standard conditions for water is a positive value, while liquids like acetone, benzene, castor oil, glycerol, just to name a few, exhibit negative changes.

\section{REFERENCES}

1. K. Takayama, "Application of shock waves to medicine", Proc. 22 $2^{\text {nd }}$ Int. Symp. on Shock Waves, G. J. Ball, R. Hillier, G. T. Roberts (eds.), v. 1., pp. 23-32, Imperial College, London, UK, 1999

2. M. A. Averkiou and R. O. Cleveland, "Modeling of an electrohydraulic lithotripter with the KZK equation", $J$. Acoust. Soc. Am., 106(1), pp. 102-112, 1999

3. T. Kodama and K. Takayama, "Dynamic behavior of bubbles during extracorporeal shock wave lithotripsy", Ultrasound in Med. Biol., 24, pp. 723-738, 1998

4. G. Jagadeesh and K. Takayama, "Novel applications of micro-shock waves in biological sciences", J. Indian Inst. Sci., 82, pp. 49-57, 2002

5. C. J. Diederich and K. Hynynen, "Ultrasound technology for hyperthermia", Ultrasound in Med. Biol., 25, pp. 871887,1999

6. R. H. Cole, Underwater explosions, Dover, New York, 1948

7. K. Nagayama, Y. Mori, M. Shimada, and M. Nakahara, "Shock Hugoniot compression curve for water up to $1 \mathrm{GPa}$ by using a compressed gas gun", J. App. Phys., 91, pp. 476-482, 2002

8. H. J. Eichler, P. Gunter, and D. W. Pohl, Laser-induced dynamic gratings, Springer-Verlag, Berlin, 1986

9. J. R. Salcedo and A. E. Siegman, "Laser induced photoacoustic grating effects in molecular crystals", IEEE J. Quantum Electron., 15, pp. 250-256, 1979

10. K. A. Nelson and M. Fayer, "Laser induced phonons: A probe of intermolecular interactions in molecular solids", $J$. Chem. Phys., 79, pp. 5202-5218, 1980 
11. T. J. Butenhoff, "Measurement of the thermal diffusivity and speed of sound of hydrothermal solutions via the laserinduced grating technique", Int. J. Thermophys., 16, pp. 1-9, 1995

12. D. C. Lamb, G. C. Lin, and A. G. Doukas, "Picosecond grating spectroscopy for characterizing the acoustic properties of biological material", App. Opt., 36, pp. 1660-1666, 1997

13. A. A. Maznev, D. J. McAulifee, A. G. Doukas, and K. A. Nelson, "Wide-band acoustic spectroscopy of biological material based on laser-induced grating technique", Ultrasound Med. Biol., 25, pp. 601-607, 1999

14. E. B. Cummings, I. A. Leyva, and H. G. Hornung, "Laser-induced thermal acoustics signals from finite beams", Appl. Opt., 35, pp. 3290-3302, 1995

15. S. Schlamp, "Laser-induced thermal acoustic velocimetry", $P h D$ Thesis, Caltech, Pasadena, California

16. P. M. Danehy, P. H. Paul, and R. L. Farrow, "Thermal-grating contributions to generate four-wave mixing in nitric oxide", J. Opt. Soc. Am., B 12, pp.1564-1576, 1995

17. B. Hemmerling, P. Radi, A. Stamapnoni-Panariello, A. Kouzov, and D. Kozlov, "Novel non-linear optical techniques for diagnostics: laser-induced gratings and two-color four-wave mixing", C. R. Acad. Sci. Paris, 2, pp. 1001-1012, 2001

18. D. W. Alderfer, G. C. Herring, P. M. Danehy, T. Mizukaki, and K. Takayama, "Sub-microsecond temperature measurement in liquid water using laser-induced thermal acoustics", Conference on Lasers and Electro-Optics, Baltimore MD, June 2003

19. B. J. Berne and R. Pecora, Dynamic light scattering, John Wiley \& Sons, New York, 1976

20. M. S. Brown and W. L. Roberts, "Single point thermometry in high-pressure, soothing premixed combustion environment", J. Ptropulsion Power, 15(1), pp. 119-127, 1999

21. R. W. Boyd, Nonlinear optics, Academic press, New York, 1992

22. R. D. Mountain, "Spectral distribution of scattered light in a simple fluid", Rev. Modern Phys., 38, pp. 205-214, 1966

23. M. Chavez, V. Sosa, and R. Tsumura, "Speed of sound in pure water", J. Acous. Soc. Am., 77, pp. 420-423, 1985. 